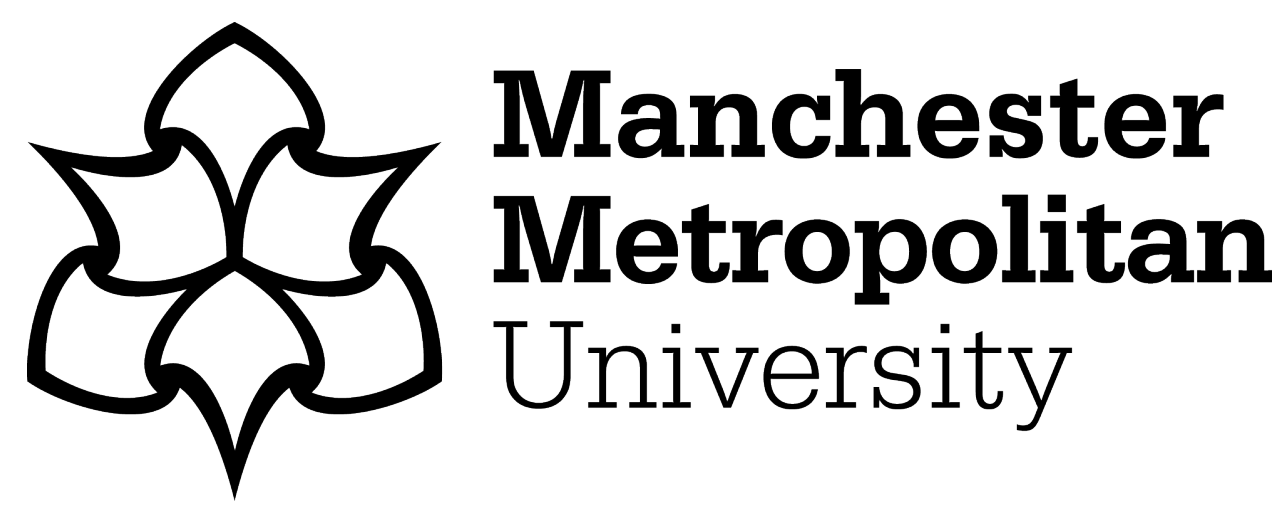

Djahel, S ORCID logoORCID: https://orcid.org/0000-0002-1286-7037, NaïtAbdesselam, F and Khokhar, A (2008) An acknowledgment-based scheme to defend against cooperative black hole attacks in optimized link state routing protocol. In: 2008 IEEE International Conference on Communications, 19 May 2008 - 23 May 2008, Beijing, China.

Downloaded from: https://e-space.mmu.ac.uk/597714/

Version: Accepted Version

Publisher: IEEE

DOI: https://doi.org/10.1109/ICC.2008.524

Please cite the published version 


\title{
An Acknowledgment-Based Scheme to Defend Against Cooperative Black Hole Attacks in Optimized Link State Routing Protocol
}

\author{
Soufine Djahel ${ }^{\mp}$, Farid Naït-Abdesselam ${ }^{\mp}$ and Ashfaq Khokhar $^{ \pm}$ \\ ${ }^{\mp}$ LIFL/IRCICA - CNRS UMR 8022 \\ University of Sciences \& Technologies of Lille, France \\ \{farid.nait-abdesselam, soufiene.djahel\}@lifl.fr \\ ${ }^{ \pm}$Electrical and Computer Engineering Department \\ University of Illinois at Chicago \\ ashfaq@ece.uic.edu
}

\begin{abstract}
In this paper, we address the problem of cooperative black hole attack, one of the major security issues in mobile ad hoc networks. The aim of this attack is to force nodes in the network to choose hostile nodes as relays to disseminate the partial topological information, thereby exploiting the functionality of the routing protocol to retain control packets. In optimized link state routing (OLSR) protocol, if a cooperative black hole attack is launched during the propagation of topology control (TC) packets, the topology information will not be disseminated to the whole network which may lead to routing disruption. In this paper, we investigate the effects of the cooperative black hole attack against OLSR, in which two colluding MPR nodes cooperate in order to disrupt the topology discovery. Then we propose an Acknowledgment based technique that overcomes the shortcomings of the OLSR protocol, and makes it less vulnerable to such attacks by identifying and then isolating malicious nodes in the network. The simulation results of the proposed scheme show high detection rate under various scenarios.
\end{abstract}

Keywords - Ad Hoc Networks, Routing Protocols, OLSR, Security, Cooperative Black Hole.

\section{INTRODUCTION}

In many applications, mobile ad hoc networks may be deployed in a hostile environment. Due to numerous constraints such as, lack of infrastructure, dynamic topology and lack of pre-established trust relationships between nodes, most of the envisioned routing protocols are vulnerable to a number of disruptive attacks. In this paper, we focus on the cooperative black hole attack which is known to be particularly challenging to defend against [5], and has been shown to be potentially damaging to a wide range of ad hoc routing protocols.

In black hole attacks, hostile nodes in general advertise availability of a fresh route without checking their routing tables. In this process, attackers always happen to be the first to reply to a route request in reactive routing context, and thus intercept the data packets being relayed and retain them. Furthermore, a special case of black hole attack, called gray hole attack, is mentioned in [4] in which only part of packets are retained while the other part is relayed. In the literature [5], [9], there are similar definitions of black and gray hole attack. However, a gray hole attack is considered as a special case of the black hole attack that has a similar impact but more difficult to detect. When this attack targets a proactive routing protocol, such as OLSR, where attackers may retain topology control packets, nodes in the entire network would be unable to get the right picture of the network topology. This will result in disrupting severely the communications as attackers will always be chosen by their neighbors as potential next hops to the destinations.
In this paper, we introduce an efficient method to detect cooperative black hole attacks. It uses an acknowledgment approach to ascertain the effective dissemination of topology control packets within the network. First, each MPR (MultiPoint Relay) node have to learn about its neighborhood up to three hops away, and then it piggybacks in its next TC message a request to an acknowledgment only from a selected subset of this set. Upon reception of the TC message, each node from this subset responds by an authenticated acknowledgment to confirm the reception of the identified TC message. By monitoring the number of missing acknowledgments, each MPR node can verify whether a cooperative black hole attack is carried out in its vicinity or not. To mitigate the impact of this attack, the detected malicious nodes will be excluded from the forwarding process by simply ignoring their Hello packets.

The rest of the paper is organized as follows. The next section summarizes the literature. Section III provides a short overview on OLSR, followed by the description of a cooperative black hole attack in section IV. In section V, we present the proposed acknowledgment based scheme to detect the misbehaving nodes. Section VI describes our simulation model and analyzes the obtained results. Finally, section VII discusses future research directions and concludes the paper.

\section{RELATED WORK}

Recently, a number of protocols and mechanisms have been proposed to secure wireless ad hoc routing. In [3], a new protocol called CONFIDANT is proposed, which aims at detecting malicious nodes by means of combined monitoring, reporting and establishment of routes that avoid misbehaving nodes. In this scheme, events have to be observable and classifiable for detection, and reputation can only be meaningful if the identity of each node is persistent, otherwise it presents vulnerabilities to spoofing attacks.

Another approach is proposed in [5] to defend against black hole attack in AODV, where they use further request and further reply packets to check the validity of a node that sends the route reply before sending the data packets. This solution assumes that malicious nodes do not exist in groups, although this is quite possible in real situations.

[6] proposes a neighborhood based method to defend against black hole attack. The solution can be briefly described as follows: once the normal path discovery procedure in the routing protocol is done, the source node sends a special control packet to request the destination to send its current neighbor set. By comparing the received neighbor sets, the source node can determine whether there is a black hole attack in the network. To mitigate the impact of the black hole attack, 
they designed a routing recovery protocol to establish the path to the correct destination.

The most effective and recent method to defend against black hole attacks is the watchdog/pathrater mechanisms[2]. This method detects misbehaving nodes acting alone by maintaining a buffer that contains recently sent packets. When a node forwards a packet, the node's watchdog ensures that the next node in the path also forwards the packet. The watchdog does this by listening to the next node promiscuously. If the next node does not forward the packet then it is termed as misbehaving. In other words, in this scheme, every packet that is overheard by the watchdog is compared with the packet in the buffer to see if there is a match. A match confirms that the packet has been successfully delivered and it is removed from the buffer. If a packet has remained in the buffer beyond the timeout period then a failure counter for the node responsible for forwarding the packet is incremented. If this counter exceeds a pre-determined threshold then the node is termed as malicious and the network is informed accordingly.

In [10], the authors present a new collusion attack model against OLSR, where the first colluding node declares in its Hello message all the 2 hop neighbors of the attacked node in order to force this latter to choose it as its unique MPR. The second attacker, acting as MPR of the first attacker, will then drop all the packets origintaed from the attacked node and passing through it. To defend against this attack, they propose to modify the standard Hello message to contain the 2 hop neighbors list. Based on this information a node can detect whether one of its neighbors has sent a false Hello message or not by looking at contradictions in the sets. However, these contradictions can no more stand if the attacker sends false neighbors set which are different from the legitimate node's 2 hop neighbors.

\section{OLSR DESCRIPTION}

The Optimized Link State Routing protocol (OLSR) [7] is a proactive routing protocol designed to work in dense networks. The main optimization of the protocol is achieved through MPRs, a set of neighbor nodes that are unique nodes in the network responsible for generating and distributing partial link state information during the flooding process, thus reducing the message overhead.

In OLSR, each node selects its MPR set from its 1 hop neighbors such that it can reach easily all its 2 hop neighbors. MPR selection process depends on the 'Willingness' value obtained from Hello message. This value indicates the willingness of a node, based on its own resources, if it is able to forward packets of other nodes. The higher the willingness is, the higher the priority the node will have to be selected as MPR. The value Will_never is chosen by nodes that are not willing to participate in the routing process. However, the value Will_always is reserved for nodes which are candidate to be selected as MPRs.

Control traffic in OLSR is exchanged through two different types of messages, namely 'Hello' and 'TC' messages. Hello messages are exchanged periodically (every $2 \mathrm{~s}$ ) between nodes to detect links between each others, to detect the identity of neighbors and to advertise MPR selections. A TC message is sent to the whole network's nodes periodically by each MPR node to declare its MPR selectors set. Information contained in TC message is used in the construction of the routing tables in each network's node.

\section{COOPERATIVE BLACK HOLE ATTACK MODEL}

In this section, we describe how two adjacent malicious nodes can launch a black hole attack in wireless ad hoc network. To ease understanding the illustration, we summarize bellow the notations and assumptions used throughout this paper. First, we assume that links are frequently symmetric. We assume also that each node holds a set of secrets for all other nodes. For example, the secret $K_{i j}$, stored within a node $\mathrm{i}$, is the secret associated to node $\mathrm{j}$. The secret is assumed to be symmetric between any pair of nodes, i.e, $K_{i j}=K_{j i}$, and are undisclosed to any other node in the network. The following

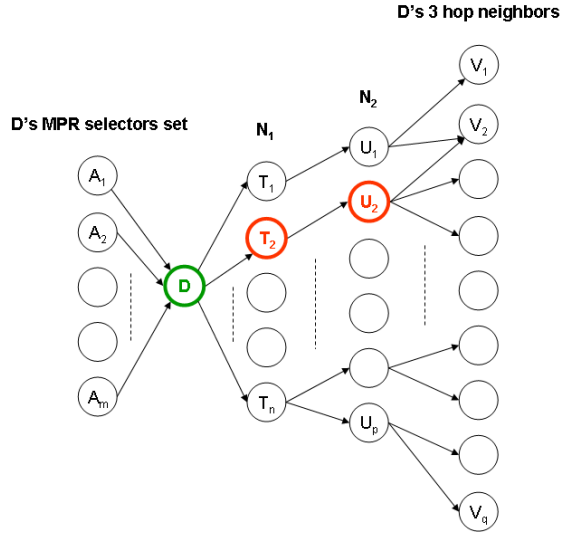

Figure 1: A cooperative attack model

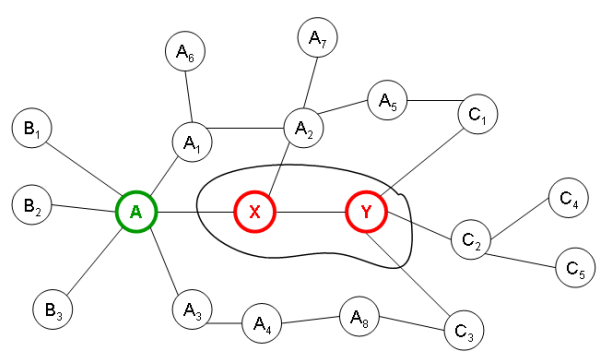

Figure 2: Cooperative black hole attack description

notations are also used to illustrate the cooperative black hole attack in OLSR.

- $N_{1}$ : the MPR set of node $D$.

- $N_{2}$ : the MPR set of $N_{1}$ 's nodes which are not in $D$ 's one hop neighborhood, i.e.,

$$
\begin{gathered}
\forall n\left(n \in N_{2} \Rightarrow \exists m\left(m \in N_{1} \wedge T\right)\right) \\
T \equiv n \in M P R \_s e t(m) \wedge n \notin n e i g h(D)
\end{gathered}
$$

- Sym $_{i}$ : the symmetric 3 hops away neighbors which have to send out an $3 h o p_{-} A C K$ packet.

- $S_{i}$ : the subset of symmetric neighbors 3 hops away reached through the node $n \in N_{2}$.

In order to launch the black hole attack in OLSR, a malicious node can force its election as MPR by maintaining constantly its Willingness field to Will_always in its HELLO message. According to the protocol, its neighbors will always select it as MPR. Using this mechanism, a malicious node can easily earn, as an MPR, a privileged position within the network. It can then exploit its rank to carry out deny of service attacks and alike. In a more sophisticated way, two colluding MPR nodes $m_{1}$ and $m_{2}$ can launch a more severe attack when the node $m_{2}$ drops all TC messages forwarded by node $m_{1}$. The attacked node, in the set of MPR selectors of $m_{1}$, can not detect this misbehavior because node $m_{2}$ is out of its radio range.

Fig. 1 shows an illustrative description of this cooperative black hole attack. Let nodes $\left\{A_{1}, \ldots, A_{m}\right\}$ be a set of nodes to be attacked and $T_{2}, U_{2}$ the attacker nodes, $\left\{T_{1}, \ldots, T_{n}\right\}$ the set of $D$ 's MPR nodes, $\left\{U_{1}, \ldots, U_{p}\right\}$ is the subset of $D$ 's 2 hop neighbors which constitutes the $N_{1}$ 's MPR nodes and $\left\{V_{1}, \ldots, V_{q}\right\}$ the set of $D$ 's 3 hop neighbors. The attack is launched as follows: node $T_{2}$ sends its HELLO message with the value of Willingness field as Will_always, i.e, all its 1 hop neighbors will choose it as an MPR. Then it chooses the node $U_{2}$ as the only MPR node to relay its TC message. By doing this, 


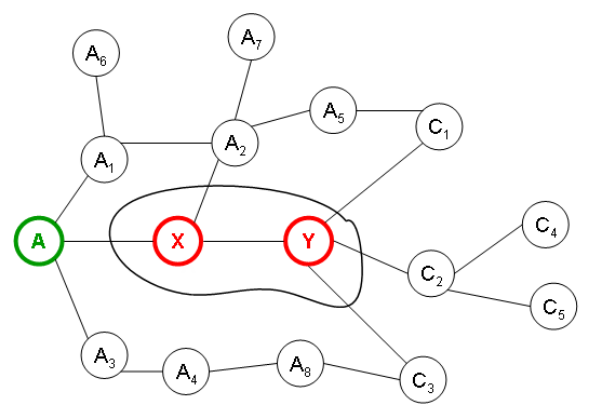

Figure 3: Topology perceived by nodes $C_{2}, C_{4}$ and $C_{5}$ after attack

node $U_{2}$ can perform the following misuses without being detected by node $D$ or any other node in its neighborhood:

- Drop all TC messages generated by node $D$, i.e, which contains addresses of nodes $\left\{A_{1}, \ldots ., A_{m}\right\}$. This will avoid the links status of $D$ 's MPR selectors to reach the nodes that are only connected to $U_{2}$. This means that some nodes in the network can not communicate with the D's MPR selectors set.

- Drop TC messages generated by node $T_{2}$ which contains the address of $D$ to prevent link information of $D$ from being disseminated to the nodes reached through it. It can also drop all TC messages passed through it.

- Modify the content of TC messages generated by node $D$ to cause inconsistent topology information dissemination.

The consequence of this attack is devastating in the presence of multiple colluding attackers around the victim node. The sender/forwarder of TC message overhears its MPR nodes transmission to ensure whether or not its sent/forwarded TC is relayed, but while the TC dropping happens out of the sender/forwarder transmission range this technique failed to detect it.

Fig. 2 shows an example of this attack. Nodes $B_{1}, B_{2}$ and $B_{3}$ constitute the MPR selectors set of node $A$. The first attacker $X$ advertises itself as having the sufficient resources to forward packets of other nodes by setting its willingness field to the highest allowed value (i.e, the value 7). According to the protocol, $X$ will be chosen as the $A$ 's MPR. Afterward, $X$ chooses the second attacker $Y$ as its unique MPR node. Thus, all TC packets generated by node $A$ and relayed by $X$ will be destroyed by $Y$.

The consequence of this attack is illustrated in Fig. 3, where nodes $C_{2}, C_{4}$ and $C_{5}$ can not build a route toward $D$ 's MPR selectors because the $D$ 's TC messages are never received (i.e, the topology information held by these nodes is incomplete).

\section{THE PROPOSED SOLUTION}

In order to deal with the cooperative black hole attack, we present an acknowledgment based scheme to mitigate the loss of topology information due to the dropping of TC messages by attackers. In addition to the original control message of OLSR, i.e, Hello and TC messages, our scheme introduces another two kinds of control packets, which are called $3 h o p_{-} A C K$ and HELLO_rep. The $3 h o p_{-} A C K$ message is used by a node to acknowledge its reception of a TC message from the neighbors 3 hops away, and the HELLO_rep message is used by a node to advertise its 2 hop neighbors to a requesting MPR node. For the request, we use one of the unused bits in the HELLO message to indicate whether the sender's MPR nodes should generate HELLO_rep packet or not. Table I shows the format of HELLO_rep message.

Our scheme requires that each MPR node should know its 3 hop neighbors set in order to be able to verify whether a malicious node, sitting out of its transmission range, misuses the transmitted

\begin{tabular}{|c|c|}
\hline MPR_node & 2_hops neighbors \\
\hline$Y$ & $C_{1} C_{2} C_{3}$ \\
$A_{2}$ & $A_{1} A_{5} A_{7}$ \\
\hline
\end{tabular}

Table I: Example of HELLO_rep message sent by node $X$

TC messages. Our scheme also requires that each MPR node has to forward all TC messages from its MPR selectors even if a message is received more than once. As such, the scheme can distinguish whether a TC message is dropped intentionally by a malicious node or by a legitimate node just because of the duplication of two TC messages.

To detect the misbehaving nodes, the sender or forwarder of the TC message maintains a list of TC packet identifiers ( $I D s)$ that have not received any $3 h o p_{-} A C K$ packet from symmetric neighbors 3 hops away. Also, a number of parameters need to be maintained, as follows:

- $A C K_{m i s s}$ : Counter for the number of $3 h o p_{-} A C K$ missed on the link $M_{1} \longleftrightarrow M_{2}$ such that $M_{1} \in N_{1}$ and $M_{2} \in N_{2}$.

- $T C_{d r o p}$ : Counter for the number of TC dropped by the direct MPR node.

- $S N T$ : List of nodes which should send out a $3 h o p_{-} A C K$ for each pair of nodes $<M_{1}, M_{2}>$.

- $\delta_{1}$ and $\delta_{2}$ : Threshold for TC packets dropped and 3hop_ACK packets missed respectively $\left(\delta_{1}<\delta_{2}\right)$.

- BlackList: List of misbehaving nodes.

When a node, say, $A$ sends or forwards a TC message to its neighbors, it monitors its MPR's transmission to verify whether MPR nodes relay the packet or not. If $A$ doesn't overhear that of MPR node $B$, it increases its $T C_{d r o p}$. If $T C_{d r o p}$ exceeds $\delta_{1}, A$ adds $B$ 's identity to the BlackList and recalculates the MPR set without node $B$. Another more complicated case is the case where node $B$ forwards the TC message sent by $A$, while it doesn't punish its MPR node $C$ which drops the TC message. To deal with this case, node $A$ increases the $A C K_{\text {miss }}$ counter for the link $B-C$ whenever the timer, which is set up to limit the reception delay of $3 h o p_{-} A C K$ packets for this link, exceeds the predefined threshold. If node $A$ receives a Hello message from $B$ in which $C$ is not in $B$ 's MPR set, while $A C K_{m i s s}$ does not exceed $\delta_{2}, A$ then adds $C$ to its Suspicious list $_{\text {i }}$; otherwise, node $A$ concludes that both nodes $B$ and $C$ are malicious and adds them to its BlackList.

Suspiciouslist is used by a MPR node $A$ to record the IDs of nodes that drop the Acknowledgment packets but move out of the forwarding path of TC messages before their Ackmiss counter reaches the threshold. As the network's topology changes rapidly, suspicious nodes can gain the same position in the forwarding path of TC messages sent/forwarded by the node $A$. $A$ then accumulates the new observations with the previous ones for a more accurate punishment.

On receiving Hello or TC message, each node deals with it as algorithms 1 and 2.

Our scheme is summarized as follows:

1) First step: Get all neighbors 3 hops away

- For each node $i$ of the $N_{1}$ set, node $D$ implicitly asking it to send its MPR set (i.e, MPR nodes of $i$ ) and the neighbors of these MPRs (i.e, 2 hops neighbors of $i$ reached through these MPRs).

- Upon reception of HELLO message in which HELLO_id is set to 1, each node of the $N_{1}$ set responds with a $H E L L O \_R e p$ packet containing the required information.

- Each MPR node sets the HELLO_id field to 1 whenever it detects a change in its MPR set or its $N_{2}$ set.

2) Second step: Sending or forwarding $T C$ message with request of acknowledgment 

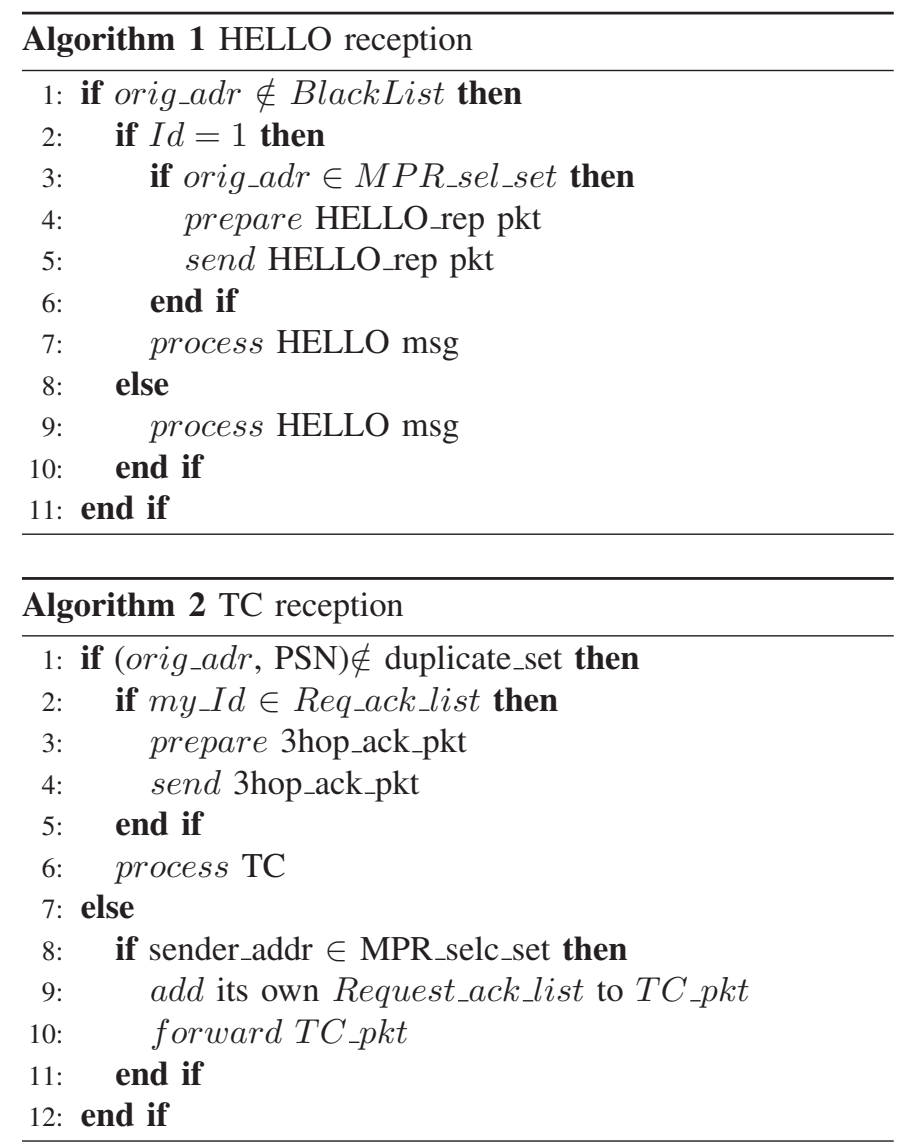

- For each node of the $N_{2}$ set, the node $D$ chooses a neighbor node such that:

$$
\left.\begin{array}{c}
\forall i \text { sym }_{i} \notin \bigcup_{\substack{j=1, \ldots, p \\
k=1, \ldots, p}}^{j \neq k}\left(S_{j} \cap S_{k}\right) \\
p=\left\|N_{2}\right\|
\end{array}\right\}(c)
$$

- When the node $D$ generates its TC message or forwards a TC message received from its MPR selectors, it inserts a request for an acknowledgment to all nodes of the set $X$, before sending it out.

$$
X=\left\{\text { Sym }_{1}, \ldots, \text { Sym }_{p}\right\}
$$

- Since PKI-based authentication is not suitable to MANETs due to the high computational complexity, we adopt an alternative solution proposed in [8] to validate the identity of $3 h o p_{-} A C K$ sender, which has moderate computation complexity and low bandwidth consumption.

\section{A. Discussion}

In Fig. 4 , node $D$ may choose node $C_{3}$, where $C_{3} \in\left(S_{Y_{1}} \cap S_{Y_{2}}\right)$, to send back a $3 h o p_{-} A C K$. In this case, node $C_{3}$ can be reached through both $Y_{1}$ and $Y_{2}$ of the set $N_{2}$. We suppose that $Y_{2}$ is a legitimate node while $Y_{1}$ is a misbehaving node. Since $D$ maintains a list of nodes that should send $3 h o p_{-} A C K$, when node $C_{3}$ receives a $T C$ message forwarded by $Y_{2}$, it sends a $3 h o p_{-} A C K$ to $D$. When $D$ gets this acknowledgment, it will delete $C_{3}$ from the $S N T$ list. Thus node $D$ believes that both nodes $Y_{1}$ and $Y_{2}$ have relayed its TC message, but actually node $Y_{1}$ did not forward it. Even node $Y_{1}$ forwards the TC message, node $C_{3}$ sends back only one $3 h o p_{-} A C K$ because it processes only the first TC message it received and ignores

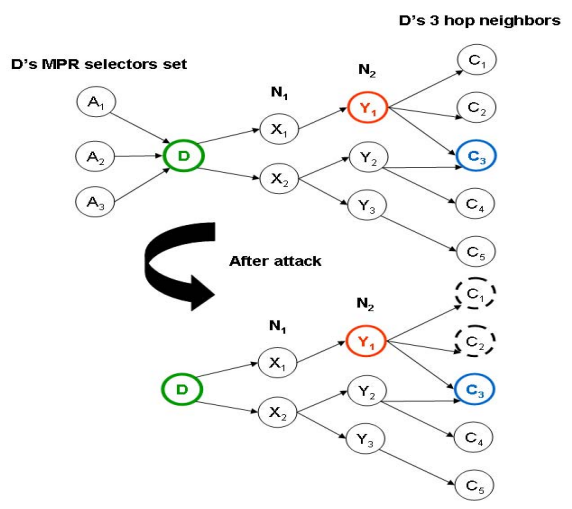

Figure 4: Topology perceived by nodes $C_{1}$ and $C_{2}$ when the condition $c$ is not satisfied

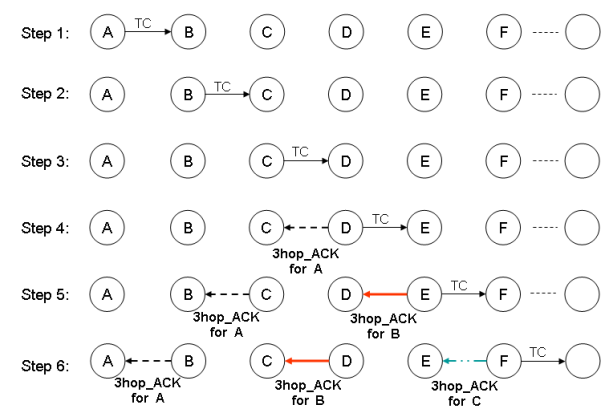

Figure 5: The 3hop_ACK scheme functioning

the later one that has the same originator address and MSN( Message Sequence Number).

\section{B. The 3hop_ACK scheme operation}

Since our scheme works at the network layer, it can be implemented as an adds on to OLSR protocol. The scheme detects individual malicious nodes by using a new type of Acknowledgment packets, named $3 h o p_{-} A C K$. The $3 h o p_{-} A C K$ packet is assigned a route of 3 hops in the opposite direction of TC packet route as shown in Fig. 5.

In Fig. 5, $A$ is assumed to be an MPR node, $B \in N_{1}, C \in N_{2}$ and $D$ the $A$ 's 3 hops neighbor which should send back the $3 h o p_{-} A C K$. When node $A$ detects changes in its $N_{1}$ or $N_{2}$ set, it sends a HELLO message with the HELLO_id field set to 1 . Upon reception of this message, each node from the set $N_{1}$ sends a HELLO_Rep packet to $A$. When $T C$ _timer expires, node $A$ generates its TC message along with a request for acknowledgment to the node $D$. This message will be forwarded by $B$ and $C$ respectively. Each receiver of this TC message checks whether its identity is included in the request list for acknowledgment; if so, it returns an authenticated $3 h o p_{-} A C K$ packet (as shown in Fig. 5). When node $A$ receives such packet, it decrypts it using the shared secret key with the anticipated sender, verifying whether the packet is sent by a legitimate node or not. Finally, node $A$ concludes that the two consecutive MPR nodes in this forwarding path behave well. This process is repeated for every quadruplet of nodes as $\langle B, C, D, E\rangle,\langle C, D, E, F\rangle$ and $\langle D, E, F, \ldots$ $>$, where the first 3 nodes are MPR nodes and the last one may not be an MPR node.

\section{Security analysis of 3hop_ACK scheme}

Our scheme is robust to a variety of attack scenarios conducted by either independent or collusive malicious nodes. In particular, we consider three typical scenarios as follows: 


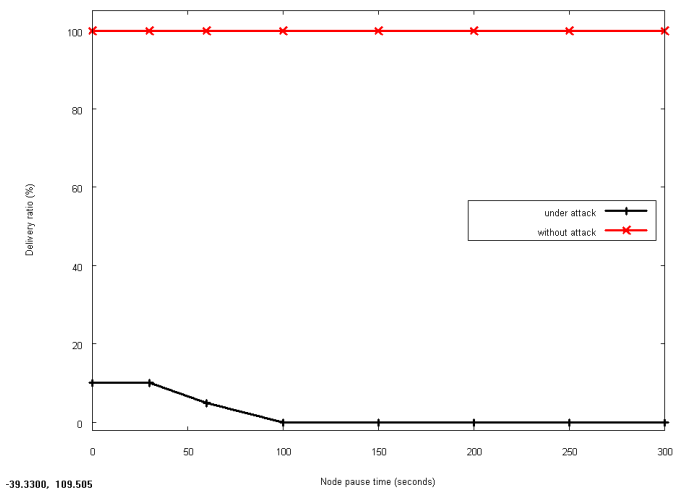

Figure 6: Delivery ratio vs. node pause time

- Scenario 1: a node in $N_{1}$ set responds with a faulty list of 2 hop neighbors in which it adds non neighbor nodes, or deletes some nodes from this list. According to our scheme, when node $D$ receives the Hello_rep from node $X$ it chooses one of the nodes listed in the 2 hop neighbors list, requiring it to send a $3 h o p_{-} A C K$. If the selected node is not an actual 2 hop neighbors of $X, D$ would not receive a $3 h o p_{-} A C K$. As a result, node $X$ will be punished.

- Scenario 2: a node in $N_{2}$ set drops the TC message and fabricates a $3 h o p_{-} A C K$ packet on behalf of the requested node. In order to fabricate this packet, the node must spoof the requested node identity and generates a valid response. But it could not compute the valid $3 h o p \_A C K$ due to the lack of knowledge on the secret key pre-shared between the requester and requested nodes.

- Scenario 3: a node $X_{2} \in N_{2}$ relays the TC message correctly but drops the $3 h o p_{-} A C K$ packet. This behavior would be detected as well, as the node $X_{1} \in N_{1}$ monitors its behavior and deletes it from the 1 hop neighbor list when the $A C K_{\text {miss }}$ counter reaches the threshold $\delta_{2}$.

\section{Simulation MODEL AND RESUlts}

This section reports the performance evaluation on our scheme by using extensive simulations conducted with the network simulator OPNET 11.5 [11]. We embedded our scheme in the implemented OLSR protocol for detecting the cooperative black hole attack. We generated random topologies with $M$ nodes over a rectangular field, where $M$ ranges from 30 to 100 . The rectangular field size is varied from $(1500 \times 1000 \mathrm{~m})$ to $(2500 \times 2000 \mathrm{~m})$. The maximum transmission range of each node is $250 \mathrm{~m}$. We run the simulation for $600 \mathrm{~s}$. Random waypoint model [1] is used as the mobility model of each node. Nodes speed is varied from $2 \mathrm{~m} / \mathrm{s}$ to $25 \mathrm{~m} / \mathrm{s}$. We change node pause time from the highest mobility (i.e, zero pause time) to 300 seconds. We use the default setting for Hello and TC messages generations as in the specification of OLSR [7]. The percentage of malicious nodes is varied from $25 \%$ (which launches 4 cooperative black hole attacks) to 40\% (which launches 20 cooperative black hole attacks). To launch the attack, the first attacker chooses randomly a victim node from its MPR selector set that has to be an MPR of the other neighbors.

\section{A. Cooperative Black hole attack simulation}

To illustrate the consequence of this attack, we generated traffic between a source node and a destination node where they are more than 3 hops away. In our scenario, this chosen destination node has as its MPR the attacked node. We define also the delivery ratio as a value of the number of received data packets to that of packets being sent by the source node. The results are shown in Fig. 6, and we observe that in the presence of the attack, the delivery ratio is $10 \%$ when node

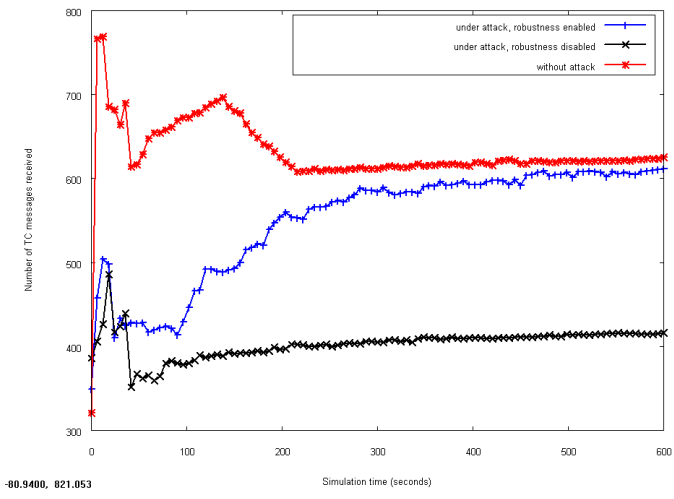

Figure 7: Comparaison of the average number of TC messages received

pause time is 0 and 30 seconds and it drops to $5 \%$ when node pause time goes to 60 seconds. The reason is that, when the destination node moves faster, it has more chances to select nodes other than the victims as MPRs and thus receives more packets; while with the lower mobility, it forces the destination node to select victim nodes as MPRs, thereby leading to fewer routes to be established between the source node and the destination.

\section{B. Performance evaluation}

We conducted extensive simulations to evaluate the performance of our scheme and show the results in Fig. 7, where the meaning of three curves are explained as follows,

- Under attack, robustness disabled: average number of receptions of each TC message generated by MPR nodes when there are attacks and our scheme is not used.

- Under attack, robustness enabled: average number of receptions of each TC messages generated by MPR nodes when there are attacks and our scheme is used.

- Without attack: average number of receptions of each TC messages generated by MPR nodes when there are no attacks.

Clearly, Fig. 7 shows that the average number of TC messages received by nodes decreases sharply when the network is under attacks and no robustness. In this case, some nodes have a partial image of the network topology because of the no reception of all TC messages generated by MPR nodes, therefore they could not establish routes to some other nodes.

When the attack is launched and our scheme is used, the average number of TC messages received is small at the beginning because the attacker node drops TC messages generated by victim nodes. This number increases at each time an attack is detected, and finally it approaches to the number of TC messages received when there is no attack. The implicit reason is that, once a malicious node is detected, the victim nodes elect a new MPR set of legitimate nodes which forwards the TC messages correctly.

Fig. 8 shows the relationship between detection rate and node pause time. We see that generally the detection rate increases as the node pause time turns to larger. Especially, when node pause time is 0 , i.e., nodes move continuously, the MPR sets of nodes change rapidly, thereby malicious nodes can drop packets and move away before its failure counter reaches the threshold. In another word, the frequency of changing of MPR set decreases with the increase of pause time, so when pause time gets larger, MPR sets is more stable over time and the performance of our scheme gets better.

In order to observe the number of false alarms triggered by our scheme, we generate a network with random topology and consists of 100 nodes, among which $40 \%$ are malicious nodes. As shown in Fig. 9, $\mathrm{Y}$ axis is the number of false alarms, $\mathrm{X}$ axis is the timeout 


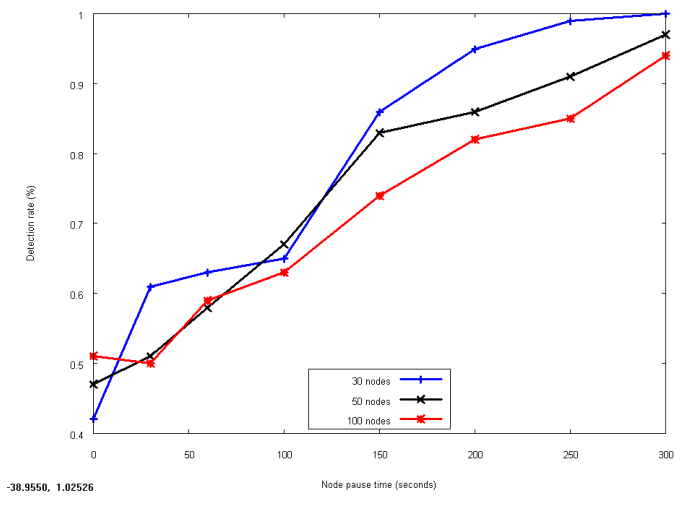

Figure 8: Detection rate vs. node pause time

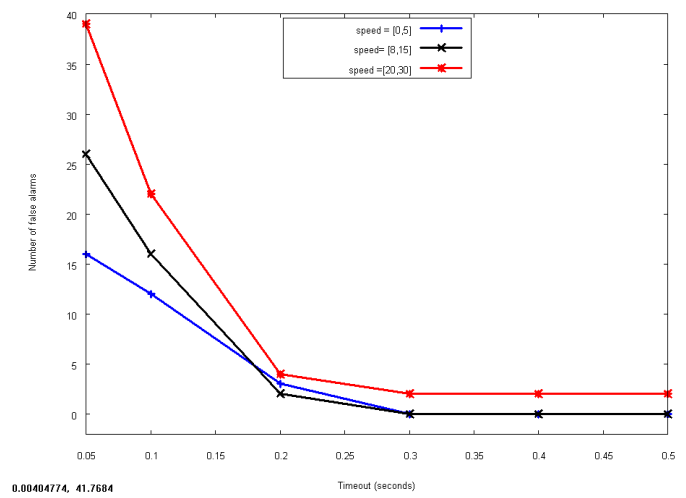

Figure 9: Number of false alarm vs. timeout value

value $\delta$, and the maximum speed of nodes varies from $0 \mathrm{~m} / \mathrm{s}$ to 30 $\mathrm{m} / \mathrm{s}$ and is divided into 3 ranges. We observed that the number of false alarms is generally proportional to the nodes speed and reduces with the increasing timeout value. We also observe that $\delta=0.3$ is the optimal threshold, after which false alarm never occur when mobility speed range is $[0,5]$ and $[8,15]$, while only 2 false alarms keep occurring for mobility speed range $[20,30]$.

\section{CONCLUSION}

Cooperative black hole attack is one of the most sophisticated attack that may result in dramatic disruption of the network performance. When launched against the OLSR protocol, a misbehaving node exploits the routing protocol's vulnerabilities to always participate in the forwarding process of TC messages, and when it colludes with another adjacent MPR node, they cooperate to prevent the dissemination of these TC messages generated by the original node in order to false the discovery of the network topology.

In this paper, we have analyzed the cooperative black hole attack in ad hoc networks and proposed an acknowledgment based scheme to detect malicious nodes and isolate them from the forwarding process. We conducted extensive simulations and the results showed high detection rate under various scenarios with negligible false positive alarms.

\section{REFERENCES}

[1] J. Broch, D.A. Maltz, D.B. Johnson, Y.C. Hu and J. Jetcheva, "A performance Comparaison of Multi-Hop Wireless Ad HOc Network Routing Protocols", in Proc. of the 4th annual ACM/IEEE international conference on Mobile computing and networking (ACM Mobicom '98), Dallas, Texas, USA, October 1998
[2] S. Marti, T.J. Giuli, K. Lai and M. Baker, "Mitigating routing misbehavior in mobile ad hoc networks", in Proc. of the 6th annual international conference on Mobile computing and networking (MOBICOM 'O0), Boston, Massachusetts, USA, August 2000.

[3] S. Buchegger and J.Y. Le Boudec, "Performance Analysis of the CONFIDANT Protocol", in Proc. of the 3rd ACM international symposium on Mobile ad hoc networking \& computing (MOBIHOC'02), Lausanne, Switzerland, June 2002.

[4] Y. Hu, A. Perrig and D. Johnson, "Ariadne: A secure On-Demand Routing Protocol for Ad Hoc Networks", in Proc. of the 8th ACM international Conference on Mobile Computing and Networking, Westin Peachtree Plaza, Atlanta, Georgia, USA, September 2002.

[5] H. Deng, W. Li and D.P. Agrawal, "Routing security in wireless ad hoc networks", in IEEE Communication Magazine, Vol. 40, No. 10, pp. 70-75, october 2002.

[6] B. Sun, Y. Guan, J. Chen and U.W. Pooch, "Detecting black- hole attack in mobile ad hoc networks", in Proc. of the 5th European Personal Mobile Communications Conference, Glasgow, UK, April 2003.

[7] T. Clausen and P. Jacquet, "Optimized Link State Routing Protocol (OLSR)". IETF RFC 3626 (Experimental), October 2003.

[8] Y.R. Tsai and S.J. Wang, "Routing security and authentication mechanism for mobile ad hoc networks", in Proc. of the 60th IEEE Vehicular Technology Conference, Los Angeles CA, USA, September 2004.

[9] W. Yu, Y. Sun and K.R.Liu, "HADOF: Defense Against Routing Disruptions in Mobile Ad Hoc Networks", in Proc. of the 24th IEEE INFOCOM, Miami, USA, March 2005.

[10] B. Kannhavong, H. Nakamaya and A. Jamalipour, "A Collusion Attack Against OLSR-based Mobile Ad Hoc Networks", in Proc. of Global Telecommunications Conference (GLOBECOM '06), San Francisco, California, USA, Nov/Dec 2006.

[11] OPNET Technologies. OPNET Modeler. http://www.opnet.com/. 